\title{
Complex oxide nanostructures by pulsed laser deposition through nanostencils
}

\author{
Cristian-Victor Cojocaru, Catalin Harnagea, Federico Rosei, ${ }^{a}$ and Alain Pignolet \\ INRS-Énergie, Matériaux et Télécommunications, Université du Québec, 1650 Boul. Lionel-Boulet, \\ Varennes, QC, J3X 1S2, Canada
}

Marc A. F. van den Boogaart and Jürgen Brugger

École Polytechnique Fédérale de Lausanne (EPFL)—Laboratoire des Microsystèmes, CH-1015 Lausanne, Switzerland

(Received 8 December 2004; accepted 18 March 2005; published online 28 April 2005)

\begin{abstract}
We achieved parallel nanoscale patterning of ferroelectric complex oxides by pulsed laser deposition through a nanostencil (i.e., through a pattern of apertures in a thin free-standing membrane). Ordered arrays of nanostructured barium titanate $\left(\mathrm{BaTiO}_{3}\right)$ were obtained onto different substrates in a single deposition step, at room temperature, replicating accurately the aperture patterns in the stencil membrane. After a postdeposition annealing treatment, x-ray diffraction pattern showed a nanocrystalline $\mathrm{BaTiO}_{3}$ structure close to the perovskite cubic phase with grains $30-35 \mathrm{~nm}$ in size. Their local ferroelectric properties were detected using piezoresponse force microscopy. (c) 2005 American Institute of Physics. [DOI: 10.1063/1.1923764]
\end{abstract}

Miniaturization of electronic devices, for instance, the nanoscale design of memories, sensors and actuators, is prompting the development of surface patterning techniques. Exploring methods to prepare and integrate complex functional materials in the standard silicon-based technology (e.g., ferroelectric materials for nonvolatile random access memories) and understanding the relationship between their functional properties and their structure and size is also an important area of focus. ${ }^{1}$ Attempts to create alternative, highresolution, and low-cost patterning processes, comparable in precision with photolithography, have led to several "unconventional" approaches. ${ }^{2-5}$ Among others, ${ }^{6}$ focused ion beam patterning, electron-beam direct writing, or nanoimprint lithography have been investigated and proposed as highresolution top-down techniques to pattern electroceramic materials. ${ }^{7}$ The common drawback of these and of traditional top-down approaches is the need for a resist or polymer process and hence, numerous chemical, thermal, and etching associated steps. Thus, the ability to fabricate arrays of functional structures with controlled size and shape, on a substrate of choice, using a minimal number of processing steps, remains an important challenge in nanotechnology. ${ }^{8}$

Recently proposed as a flexible method to control the parallel patterning of nanostructures, the nanostenciling approach offers high versatility in combining various functional materials and different substrates by reducing the number of processing operations with respect to resist-based lithography. ${ }^{9}$ However, to date, deposition through a nanostencil has been reported only for simple metals. ${ }^{10-14}$ The miniature shadow masks used are reusable, and protective coatings have been tested to increase their lifetime. ${ }^{15}$

The central issue addressed in this letter is the study of nanostructuring and patterning of complex functional materials (such as perovskite ferroelectric oxides). ${ }^{16}$ We report here on nanostructure patterning of barium titanate $\left(\mathrm{BaTiO}_{3}\right)$ on silicon and strontium titanate $\mathrm{SrTiO}_{3}$ (100) obtained via nanostenciling.

\footnotetext{
${ }^{a)}$ Electronic mail: rosei@emt.inrs.ca
}

A first set of experiments was conducted with stencil masks made of silicon nitride nanosieves with circular holes, fabricated by a combination of laser interference lithography and silicon micromachining. ${ }^{17}$ Hexagonal arrays of pores (down to $300 \mathrm{~nm}$ in diameter) were patterned and transferred into free-standing low-stress (LS-SiN) membranes, prepared on single-crystalline (100) silicon wafers. Finally, the wafers were diced into square pieces of $5 \times 5 \mathrm{~mm}^{2}$ (stencil's dimension). Figure 1(a) displays a scanning electron micrograph detail from a LS-SiN nanosieve with pores of $300 \mathrm{~nm}$ in diameter and a $1.6 \mu \mathrm{m}$ pitch. ${ }^{18,19}$

The miniature shadow masks were mechanically attached and temporarily fixed onto the substrate ${ }^{20}$ and the assembly substrate stencil was mounted in a pulsed laser deposition (PLD) chamber, in front of a rotating target. $\mathrm{BaTiO}_{3}$, an oxide with perovskite crystal structure used in capacitors and piezoelectric devices, was chosen as material to be tested. ${ }^{21}$ A $\mathrm{KrF}$ excimer laser $(\lambda=248 \mathrm{~nm}$, pulse duration $=14 \mathrm{~ns}$ ) was employed for ablation with an incidence angle of the laser beam on the target of $45^{\circ}$ and laser fluence set at $2 \mathrm{~J} / \mathrm{cm}^{2}$. Deposition was carried out in vacuum at $5 \times 10^{-5}$ mbar at room temperature, with a laser repetition rate of $5 \mathrm{~Hz}$.

The films were investigated by atomic force microscopy (AFM), scanning electron microscopy (SEM) and x-ray dif-
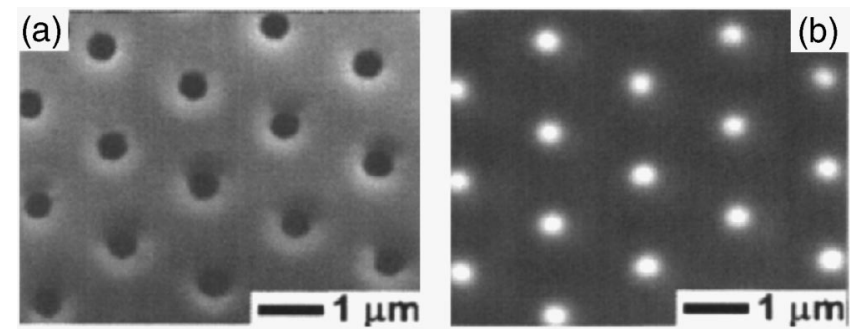

FIG. 1. (a) SEM image of a LS-SiN nanosieve with $300 \mathrm{~nm}$ diameter pores and $1.6 \mu \mathrm{m}$ pitch (b) Tapping mode AFM image (height) of as-deposited $\mathrm{BaTiO}_{3}$ dots on $\mathrm{Si}(100)$ by PLD at room temperature through the periodic arrays of apertures made in the LS-SiN membrane. 

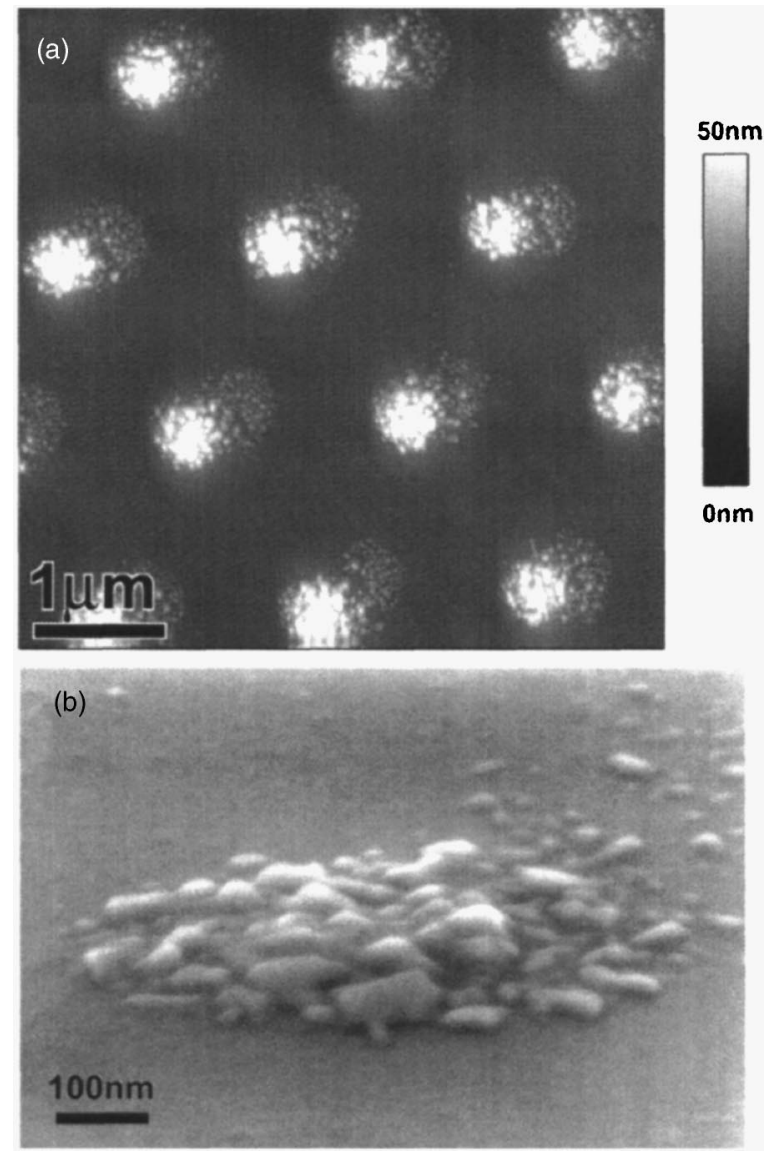

FIG. 2. (a) $5 \times 5 \mu \mathrm{m}^{2}$ contact mode $\mathrm{AFM}$ topography image of $\mathrm{BaTiO}_{3}$ structures on $\mathrm{Si}(100)$ after annealing at $650{ }^{\circ} \mathrm{C}$ for $1 \mathrm{~h}$ in $\mathrm{O}_{2}$ flow. (b) SEM micrograph detail of an individual $\mathrm{BaTiO}_{3}$ structure.

fraction (XRD) after annealing at temperatures in the range $650-900{ }^{\circ} \mathrm{C}$. AFM and piezoresponse force microscopy (PFM) measurements ${ }^{22}$ were performed using a commercial atomic force microscope (DI-EnviroScope, Veeco Instruments). ${ }^{23}$

Rapid fabrication of ordered nanostructures of $\mathrm{BaTiO}_{3}$ structures was achieved in a single deposition step. ${ }^{24}$ Using a first batch of stencils with circular holes, ordered hexagonal arrays of dot-like structures were grown over the whole sieve areas $(1 \mathrm{~mm}$ in length $\times 100 \mu \mathrm{m}$ in width). Figure 1 (b) shows the topography (height) of the well-ordered structures observed by AFM in tapping mode. The as-deposited $\mathrm{BaTiO}_{3}$ nanostructures grown on $\mathrm{Si}(100)$ have a dome shape with a height $(h)$ of $50 \mathrm{~nm}$ and a width $(w)$ of $400 \mathrm{~nm}$ [full width at half maximum (FWHM)]. ${ }^{25}$

A postdeposition annealing treatment, at temperatures of $650{ }^{\circ} \mathrm{C}, 800^{\circ} \mathrm{C}$, and $900{ }^{\circ} \mathrm{C}$ for $1 \mathrm{~h}$ in $\mathrm{O}_{2}$ flow, was used to crystallize the structures. After annealing, the aspect of the dots changed from an amorphous dome shape to an agglomeration of crystallites for each dot. The annealed samples were investigated by AFM using different cantilevers/tips both in contact and in tapping mode. A $5 \times 5 \mu \mathrm{m}^{2}$ AFM topographic image (contact mode) of the annealed $\mathrm{BaTiO}_{3}$ structures on the $\mathrm{Si}(100)$ substrate is presented in Fig. 2(a). The tail-like shapes of the features in the AFM micrograph might be caused by a slight off-axis geometry of the PLD target with respect to the stencil/substrate. The SEM micrograph detail shown in Fig. 2(b) illustrates the $\mathrm{BaTiO}_{3}$ structure's shape evolution after annealing. ${ }^{26}$

Downloaded 28 Apr 2005 to 207.162.24.31. Redistribution subject
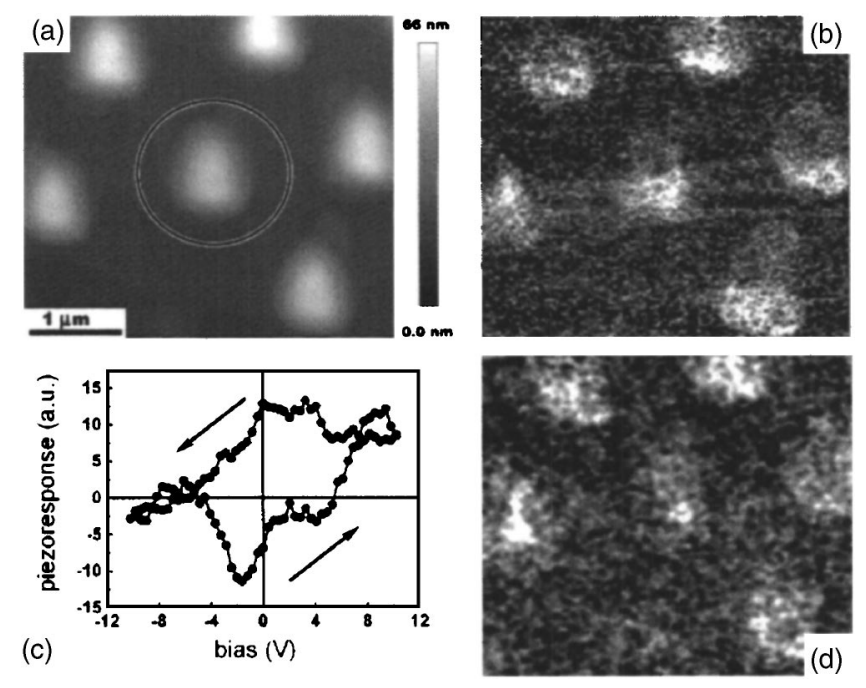

FIG. 3. AFM and PFM investigation of individual $\mathrm{BaTiO}_{3}$ structures on a Nb-doped 100-oriented $\mathrm{SrTiO}_{3}$ substrate (a) topography, (b) ferroelectric domain structure before switching, (c) switching piezoresponse hysteresis loop, and (d) ferroelectric domain structure after switching.

The continuous films and the patterned samples were analyzed by XRD at grazing-angle incidence $\left(\omega=1^{\circ}\right)$ [X-Pert Pro (PANalytical) Diffractometer]. Experimental data (not shown here) obtained for the patterned ordered structures and for the surrounding film area show the presence of a polycrystalline phase very similar to the perovskite cubic phase with grains $30-35 \mathrm{~nm}$ in size (using the Scherrer formula). It is known that polycrystalline $\mathrm{BaTiO}_{3}$ thin films with fine grain size (below $100 \mathrm{~nm}$ ) could exhibit weaker ferroelectric properties than larger grained films/ ceramics or bulk single crystals. ${ }^{27}$ However, using long integration times for the lock-in amplifier, we recorded piezoelectric hysteresis loops from individual structures, as shown in Fig. 3. The ferroelectric domain structure of the $\mathrm{BaTiO}_{3}$ nanostructures was probed by PFM. Figure 3(a) presents the AFM topographical image of $\mathrm{BaTiO}_{3}$ patterned on a Nbdoped (100) $\mathrm{SrTiO}_{3}$ substrate together with the piezoresponse domain image simultaneously recorded [Fig. 3(b)]. The bright regions reveal that the dots predominantly have a spontaneous polarization oriented downward. The PFM signal was very weak, which is attributed to the reduced tetragonality of the $\mathrm{BaTiO}_{3}$ unit cell caused by the fine grain size of the nanostructures $(\sim 30 \mathrm{~nm})$. However, the piezoresponse hysteresis loop [Fig. 3(c)] reveals that the spontaneous polarization of the dots can still be switched [Fig. 3(d)] and that the nanostructures retain ferroelectricity. This effect proves that not only the chemical composition and the crystal structure but also the functionality of the complex oxide nanostructures deposited through the nanostencils are conserved and that, in this case, ferroelectricity in $\mathrm{BaTiO}_{3}$ still exists even at this small grain size. These results demonstrate the power of the nanostenciling approach to investigate the growth and properties of nanostructured complex materials, and to study possible size effects on their functional properties. Within the frame of the present investigation, a detailed study of the effect of the annealing temperatures ${ }^{28}$ and of the effects of the size on the well-defined $\mathrm{BaTiO}_{3}$ pattern of nanostructures obtained by nanostenciling on different substrates is currently under way. This will lead to the optimization of the whole deposition and patterning process of the to AlP license or copyright, see http://apl.aip.org/apl/copyright.jsp 
complex perovskite $\mathrm{BaTiO}_{3}$, yielding the parameters needed to obtain nanoscale functionality.

In summary, PLD of complex materials through a nanostencil is demonstrated, offering a simple method to fabricate well-ordered structures from complex functional materials (e.g., $\mathrm{BaTiO}_{3}$ ) under high-vacuum or ultrahigh vacuum conditions. Annealing of the as-deposited structures at temperatures below $900{ }^{\circ} \mathrm{C}$ yields a polycrystalline phase with very fine grains very similar to the cubic phase of $\mathrm{BaTiO}_{3}$. Ferroelectric switching in these nanostructures was shown by PFM, prompting a more detailed structural analysis. Several issues remain to be investigated, such as the large-scale uniformity of the deposited nanostructures across the substrate and the dependence of their functional properties with size.

Two of the authors (F. R.) and (A. P.) acknowledge financial support from the Natural Science and Engineering Research Council (NSERC) of Canada, NanoQuebec, and INRS startup funds. One of those authors (F. R.) is grateful to FQRNT and the Canada Research Chairs program for salary support. Another author (M. A. F. v. d. B.) acknowledges financial support from the Swiss Innovation Promotion Agency KTI/CTI, Programme TopNano 21.

${ }^{1}$ C. H. Ahn, K. M. Rabe, and J.-M. Triscone, Science 303, 488 (2004).

${ }^{2}$ Y. Xia, J. A. Rogers, K. E. Paul, and G. M. Whitesides, Chem. Rev. (Washington, D.C.) 99, 1823 (1999).

${ }^{3}$ S. B. Clendenning, S. Aouba, M. S. Rayat, D. Grozea, J. B. Sorge, P. M. Brodersen, R. N. Sodhi, Z. H. Lu, C. M. Yip, M. R. Freeman, H. E. Ruda, and I. Manners, Adv. Mater. (Weinheim, Ger.) 16, 215 (2004).

${ }^{4}$ C. M. Sotomayor Torres, S. Zankovych, J. Seekamp, A. P. Kam, C. C. Cedeno, T. Hoffmann, J. Ahopelto, F. Reuther, K. Pfeiffer, G. Bleidiessel, G. Gruetzner, M. V. Maximov, and B. Heidari, Mater. Sci. Eng., C 23, 23 (2003).

${ }^{5}$ J. H. Weaver and V. N. Antonov, Surf. Sci. 557, 1 (2004).

${ }^{6}$ I. Szafraniak, C. Harnagea, R. Scholz, S. Bhattacharyya, D. Hesse, and M. Alexe, Appl. Phys. Lett. 83, 2211 (2003).

${ }^{7}$ M. Alexe, C. Harnagea, and D. Hesse, J. Electroceram. 12, 69 (2004).

${ }^{8}$ F. Rosei, J. Phys.: Condens. Matter 16, S1373 (2004).

${ }^{9}$ J. Brugger, J. W. Berenschot, S. Kuiper, W. Nijdam, B. Otter, and M. Elwenspoek, Microelectron. Eng. 53, 403 (2000).

${ }^{10}$ M. M. Deshmukh, D. C. Ralph, M. Thomas, and J. Silcox, Appl. Phys. Lett. 75, 1631 (1999).

${ }^{11}$ J. Kohler, M. Albrecht, C. R. Musil, and E. Bucher, Physica E (Amsterdam) 4, 196 (1999).
${ }^{12}$ G. M. Kim, M. A. F. van den Boogaart, and J. Brugger, Microelectron. Eng. 67, 609 (2003).

${ }^{13}$ E. A. Speets, B. J. Ravoo, F. J. G. Roesthuis, F. Vroegindeweij, D. H. A. Blank, and D. N. Reinhoudt, Nano Lett. 4, 841 (2004).

${ }^{14}$ F. Vroegindeweij, E. A. Speets, J. A. J. Steen, J. Brugger, and D. H. A. Blank, Appl. Phys. A: Mater. Sci. Process. 79, 743 (2004).

${ }^{15}$ M. Kolbel, R. W. Tjerkstra, G. M. Kim, J. Brugger, C. J. M. van Rijn, W. Nijdam, J. Huskens, and D. N. Reinhoudt, Nano Lett. 2, 1339 (2002).

${ }^{16}$ M. E. Lines and A. M. Glass, Principles and Applications of Ferroelectrics and Related Materials, (Clarendon, Oxford, 1977).

${ }^{17}$ Nanostencil samples were provided by Aquamarijn Microfiltration, B. V., Netherlands.

${ }^{18} \mathrm{~A}$ second batch of stencils $\left(1 \times 1 \mathrm{in.}^{2}\right.$, with 65 perforated LS-SiN membranes, $200 \mathrm{~nm}$ thick) was used in a further set of experiments giving the possibility of testing the deposition process through features with various integrated sizes (nm and $\mu \mathrm{m}$ ranges) and shapes. Deep ultravioletmicroelectromechanical system stencils were fabricated at EPFLMicrosystems Laboratory Lausanne (LMIS1), Switzerland.

${ }^{19}$ M. A. F. van den Boogaart, G. M. Kim, R. Pellens, J.-P. van den Heuvel, and J. Brugger, J. Vac. Sci. Technol. B 22, 3174 (2004).

${ }^{20}$ Different materials, such as $\mathrm{Si}(100)$, platinum- $(\mathrm{Pt})$ coated silicon, or $0.1 \%$ Nb-doped $\mathrm{SrTiO}_{3}$ (100)-oriented, have been used as substrates.

${ }^{21}$ The target used was made out of a dense stoichiometric $\mathrm{BaTiO}_{3}$ ceramic. A set of $\mathrm{BaTiO}_{3}$ thin films (20-200 nm thick) was grown by PLD on silicon (100) substrates to serve as a control experiment and to optimize the growth parameters.

${ }^{22} \mathrm{C}$. Harnagea and A. Pignolet, in Nanoscale Characterization of Ferroelectric Materials-Scanning Probe Microscopy Approach, edited by M. Alexe and A. Gruverman (Springer, Berlin, 2004), Chap. 2, pp. 47-64.

${ }^{23}$ For PFM, a computer-controlled lock-in amplifier (Signal Recovery Model 7265) was connected to the AFM via a Signal Access Module. To apply the voltage to the sample, we used conductive cantilevers CSC11 A (spring constant $0.6 \mathrm{~N} / \mathrm{m}$ ) with semiconductor $\mathrm{W}_{2} \mathrm{C}$ from Micromash. An ac testing voltage of $0.5 \mathrm{~V}$ was applied between the tip and the conductive substrate on which the structures were deposited. Hysteresis measurements were obtained using an auxiliary digital to analog converter of the lock-in amplifier, by sweeping the voltage from maximum to minimum values.

${ }^{24}$ The parameters optimized for PLD of $100 \mathrm{~nm}$ thick $\mathrm{BaTiO}_{3}$ layers were used for growth through the stencils.

${ }^{25} \mathrm{~A}$ broadening of the bottom width of the structures, caused by the shadow effect of the mask, occurs when the stencil is not pressed tightly enough against the substrate. Structures as small as $250 \mathrm{~nm}$ in width (FWHM) were obtained after consecutive depositions through the same stencil.

${ }^{26}$ Since the structures were grown on $\mathrm{Si}$ substrates covered with a native amorphous $\mathrm{SiO}_{2}$ layer, we do not expect to see an oriented growth of the $\mathrm{BaTiO}_{3}$ structures, even after annealing.

${ }^{27}$ R. Waser, Integr. Ferroelectr. 15, 39 (1997).

${ }^{28}$ K. M. Ring and K. L. Kavanagh, J. Appl. Phys. 94, 5982 (2003). 